\title{
Informations- und Aufklärungspflichten des Arztes
}

\author{
Dr. Thorsten A. Quiel - Rechtsanwalt, Fachanwalt für Medizinrecht, Bonn
}

Am 26. Februar 2013 ist das Gesetz zur Verbesserung der Rechte von Patientinnen und Patienten in Kraft getreten. Dr. Thorsten A. Quiel, Fachanwalt für Medizinrecht, fasst die Kernaussagen für Sie zusammen.

Das Gesetz zur Verbesserung der Rechte von Patientinnen und Patienten, kurz „Patientenrechtegesetz“, enthält unter anderem Regelungen zu den Informationsund Aufklärungspflichten des behandelnden Arztes und zudem auch zur Einwilligung des Patienten als Voraussetzung für eine gerechtfertigte ärztliche Behandlung.

\section{Informieren über Therapie, weitere Maßnahmen und Kosten}

Nach $₫ 630$ c Abs. 2-4 BGB muss der behandelnde Arzt den Patienten in verständlicher Form zu Beginn der Behandlung und, soweit erforderlich, in deren Verlauf über sämtliche für die Behandlung wesentlichen Umstände informieren, insbesondere die Diagnose, die voraussichtliche gesundheitliche Entwicklung, die Therapie und die zu und nach der Therapie zu ergreifenden Maßnahmen.

Sollten für den Arzt Umstände erkennbar sein, die die Annahme eines Behandlungsfehlers begründen, hat er den Patienten über diese auf Nachfrage oder zur Abwendung gesundheitlicher Gefahren $\mathrm{zu}$ informieren.
Ist der Arzt in Kenntnis darüber, dass eine vollständige Übernahme der Behandlungskosten durch einen Dritten nicht gesichert ist oder ergeben sich dafür hinreichende Anhaltspunkte, muss er den Patienten vor Beginn der Behandlung über deren voraussichtliche Kosten schriftlich informieren.

Der Information bedarf es generell dann nicht, wenn besondere Umstände sie entbehrlich machen, insbesondere wenn die Behandlung unaufschiebbar ist oder der Patient auf die Information verzichtet hat.

\section{Patient oder Berechtigter müssen einwilligen}

Vor Beginn der Behandlung ist der Arzt verpflichtet, die Einwilligung des Patienten einzuholen ( $\$ 630 \mathrm{~d}$ BGB). Ist dieser einwilligungsunfähig, kommt es auf die Einwilligung eines hierzu Berechtigten an, soweit nicht eine Patientenverfügung die Maßnahme gestattet oder untersagt. Kann eine Einwilligung zu einer unaufschiebbaren Maßnahme nicht rechtzeitig eingeholt werden, reicht eine Einwilligung auf der Grundlage des mutmaßlichen Willens des Patienten.

Eine einmal erteilte Einwilligung kann jederzeit und ohne Angabe von Gründen formlos widerrufen werden. Ihre Wirkung entfällt damit natürlich nicht für die Vergangenheit.

\section{Aufklären über Folgen und Risiken}

Der Arzt ist laut $\$ 630$ e Abs. 1-5 BGB dazu verpflichtet, den Patienten über

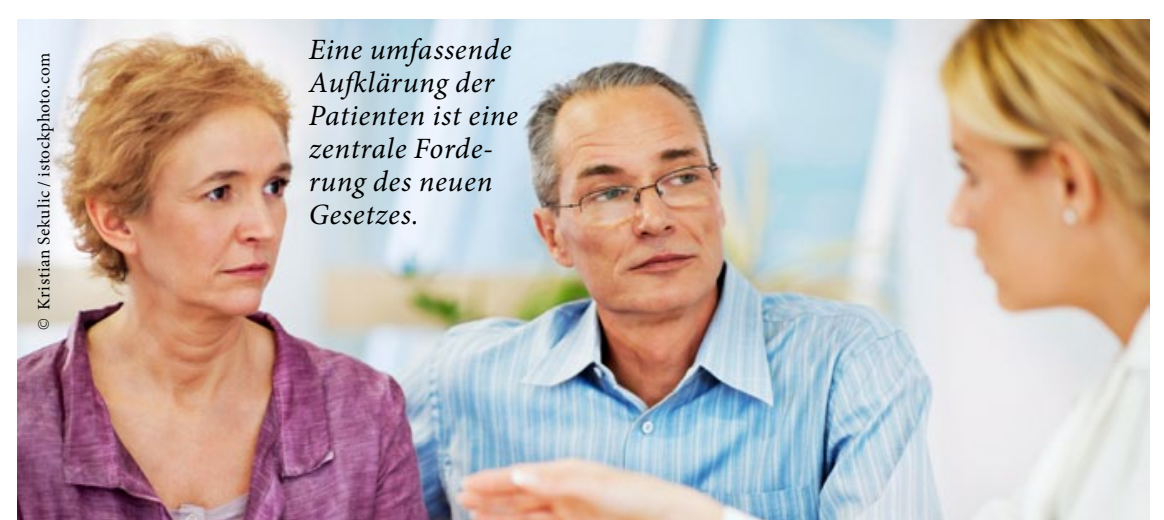

sämtliche für die Einwilligung wesentlichen Umstände vor der Behandlung aufzuklären, also insbesondere über Art, Umfang, Durchführung, zu erwartende Folgen und Risiken der Maßnahme sowie ihre Notwendigkeit, Dringlichkeit, Eignung und Erfolgsaussichten im Hinblick auf die Diagnose oder die Therapie.

Im Rahmen der Aufklärung ist auch auf Alternativen zur Maßnahme hinzuweisen, wenn mehrere medizinisch gleichermaßen indizierte und übliche $\mathrm{Me}$ thoden zu wesentlich unterschiedlichen Belastungen, Risiken oder Heilungschancen führen können, also „echte“ Behandlungsalternativen bestehen.

Die Aufklärung muss in einer für den Patienten verständlichen Form, rechtzeitig vor der Behandlung mündlich durch den behandelnden Arzt oder durch eine Person erfolgen, die über die zur Durchführung der Maßnahme notwendige Ausbildung verfügt. Ergänzend kann auf Unterlagen Bezug genommen werden, die dem Patienten ausgehändigt werden. Abschriften von Unterlagen, die er im Zusammenhang mit der Aufklärung oder Einwilligung unterzeichnet hat, müssen ihm ausgehändigt werden.

Einer Aufklärung bedarf es nicht, wenn diese ausnahmsweise aufgrund besonderer Umstände entbehrlich ist, etwa wenn die Maßnahme unaufschiebbar ist oder der Patient auf die Aufklärung ausdrücklich verzichtet.

Bei fehlender Einwilligungsfähigkeit des Patienten ist die Einwilligung eines hierzu Berechtigten einzuholen, der dann entsprechend aufzuklären ist. In diesem Falle sind die wesentlichen Umstände zur Aufklärung auch dem Patienten entsprechend seinem Verständnis zu erläutern, soweit dieser aufgrund seines Entwicklungsstandes und seiner Verständigungsmöglichkeiten in der Lage ist, die Erläuterungen aufzunehmen, und soweit dies seinem Wohl nicht zuwider läuft. 